\title{
Estudo morfológico do aluminato de cálcio dopado com cério sintetizado por método de combustão em solução
}

\author{
Morphologic study of calcium aluminate doped with cerium synthesized by \\ the method of combustion in solution
}

Raquel Jovita Santos ${ }^{1}$, Valéria Rejane Silva Brito; Paulo Neilson Marques dos Anjos ${ }^{1}$, Severino Alves Junior ${ }^{2}$

\section{RESUMO}

O aluminato de cálcio é um material utilizado em formações estruturais, quando dopado com metais terras raras funciona como matriz para constituição de material luminescente. A morfologia da matriz influencia na cor e luminescência do íon terra rara. Portanto este trabalho investiga a composição da matriz aluminato de cálcio obtido pelo método de combustão em solução. O material é preparado através da mistura das soluções dos nitratos de cálcio e alumínio em mistura com as soluções do combustível e do dopante íon cério. Foram preparados aluminatos com dois tipos de combustíveis, a glicina e a sacarose, em proporções de acordo a reação redox de 1,0 e 2,5 correspondentes à estequiometria e acima da estequiométrica, respectivamente. A morfologia do material foi analisada, após calcinação do precursor a $800{ }^{\circ} \mathrm{C}$, por TEM e MEV observando a homogeneidade e constituição do material e por DRX obtendo composições com misturas de fases de $\mathrm{CaAl}_{2} \mathrm{O}_{4}$ e $\mathrm{Ca}_{2} \mathrm{Al}_{3} \mathrm{O}_{6}$, sendo a primeira fase a predominante nas amostras.

Palavras-chave: Aluminato de cálcio, Difração de raios-X, Microscopia eletrônica de transmissão.

\begin{abstract}
Calcium aluminate is a material used in structural formations, when doped with rare earth metal ions function as a matrix for the constitution of luminescent material. The morphology of the matrix influences the color and luminescence of the rare earth. This work investigated the composition of the calcium aluminate matrix obtained by the method of combustion in solution. The material was prepared by mixing the solutions of the calcium and aluminum nitrates and addiction the solutions of the fuel and the doping element cerium as nitrate. The material was prepared with two types of fuels the glycine and sucrose in proportions to the redox reaction of 1.0 and 2.5 , corresponding to stoichiometry ratios between nitrates and the fuels. The material was analyzed after the calcination of the precursor at $800^{\circ} \mathrm{C}$ by microscopy TEM and SEM getting features of homogeneity and crystallinity and by XRD obtaining compositions with phase mixtures of $\mathrm{CaAl}_{2} \mathrm{O}_{4}$ and $\mathrm{Ca}_{2} \mathrm{Al}_{3} \mathrm{O}_{6}$, the former being predominant in the samples.
\end{abstract}

Keywords: Calcium aluminate, X-ray diffraction, Transmission electronic microscopy

\footnotetext{
'Universidade Estadual de Santa Cruz - Departamento de Ciências Exatas e Tecnológicas - Laboratório de Pesquisa e Inovação de Materiais Avançados - Campus Soane Nazaré de Andrade - Ilhéus (BA) - Brasil

2Universidade Federal de Pernambuco - Departamento de Química Fundamental - Laboratório de Terras Raras -- Recife (PE) - Brasil. Autor correspondente: Paulo Neilson M. dos Anjos - Universidade Estadual de Santa Cruz - Departamento de Ciências Exatas e Tecnológicas - Laboratório de Pesquisa e Inovação de Materiais Avançados - Campus Soane Nazaré de Andrade - Rodovia Jorge Amado, Km 16 - CEP $45662-900$ - Ilhéus (BA) - Brasil Email: pauloneilson@uesc.br

Recebido: 29/09/2016 Revisado: 22/02/2017
} 


\section{INTRODUÇÃO}

O aluminato de cálcio é usado como cerâmica estrutural, refratário e acrescentado ao cimento para conferir propriedades de resistência mecânica em altas temperaturas ${ }^{(1,2)}$. Os aluminatos possuem dentre as diversas conformações estruturais uma conhecida como espinélio, com composição na proporção de 1:2 de metais e fórmula molecular $\mathrm{MAl}_{2} \mathrm{O}_{4},(\mathrm{M}=\mathrm{Ba}, \mathrm{Ca}, \mathrm{Sr} . .$.$) .$ Essa estrutura é derivada de cubos com esferas nas arestas com a unidade celular cúbica de face centrada dobrada de 8 células contendo espaços de 32 octaedros e 64 tetraedros como sítios de metais. $\mathrm{Na}$ estrutura espinélio $50 \%$ dos espaços octaédricos são designados à posição do alumínio e um oitavo dos espaços tetraédricos são sítios do $\mathrm{M}^{+2}$, como demonstra a Fig. 1 .

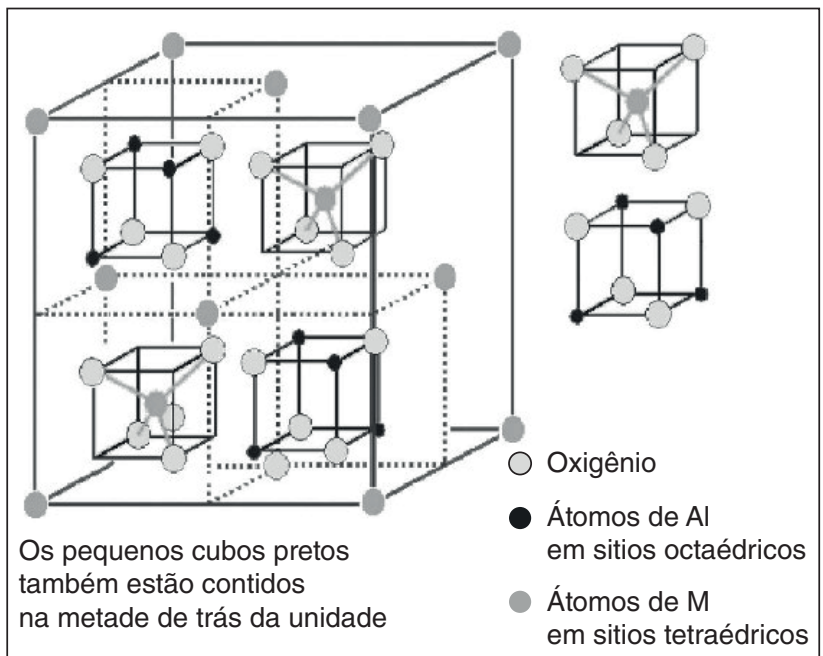

Figura 1: Estrutura geral para o espinélio de aluminatos de metal. (PATIL, et. al., 2008)

Devido à sua conformação estrutural, a estrutura cristalina do aluminato, quando dopado com metais de grande dimensão, como os lantanídeos, não é deformada tão facilmente em diversos processos de síntese ${ }^{(1)}$ e pela dopagem com diferentes concentrações dos lantanídeos é obtido o fenômeno de longa permanência da fosforescência, ou seja, a luminescência que permanece por um longo tempo (de segundos para horas) ao término da fonte de excitação. ${ }^{(3)}$ Essa propriedade óptica dos aluminatos dopados com terras raras é investigada desde 1996, com o primeiro material, $\mathrm{SrAl}_{2} \mathrm{O}_{4}: \mathrm{Eu}^{2+}, \mathrm{Dy}^{3+}$ caracterizado por Matsuzawa ${ }^{4}$ (1996, p. 170 apud Liu, 2016), apresentando fosforescência que perdura mais de 30 horas $^{(3)}$. Atualmente os materiais $\mathrm{SrAl}_{2} \mathrm{O}_{4}: \mathrm{Eu}^{2+}, \mathrm{Nd}^{3+}$ e $\mathrm{SrAl}_{2} \mathrm{O}_{4}: \mathrm{Eu}^{2+}, \mathrm{Dy}^{3+}$, que possuem luminescência no azul e verde respectivamente, estão comercialmente disponíveis ${ }^{(5)}$. Estudos recentes têm investigado o comportamento de emissão dos íons $\mathrm{Ce}^{3+}$, onde a transição $4 \mathrm{f}-5 \mathrm{~d}$ possui diferentes emissões eletromagnéticas do visível, pois dependem da intensidade do campo cristalino da matriz. Fósforos de longa permanência do $\mathrm{Ce}^{3+}$ são relatados por $\mathrm{Kodama}^{(5)}$ et al (2000) em Ca $\mathrm{Al}_{2} \mathrm{SiO}_{7} \mathrm{e} \mathrm{CaYAl}_{3} \mathrm{O}_{7}$, por Madhukumar ${ }^{(6)}$ et al (2016). em matriz de $\mathrm{CaAl}_{2} \mathrm{O}_{4}$ e por $\mathrm{Chen}^{(7)}$ et al (2012). em matriz de
$\mathrm{Ca}_{2} \mathrm{Al}_{3} \mathrm{O}_{6}$. Fósforos luminescentes de longa permanência possuem aplicações em detectores de radiação ${ }^{(8)}$, sensores ópticos ${ }^{(9)}$, sinais de trânsito, decoração de interiores, iluminação de segurança e LEDS de televisores ${ }^{(3)}$. Com extensão para a área médica em diagnóstico de câncer, terapia fotodinâmica e imagens in vivo ${ }^{(3)}$.

A composição é importante para determinação da cor de emissão referente ao espectro visível e características de resistências dos materiais. Com vistas a melhoramentos estruturais, os diferentes métodos de síntese visam à obtenção de materiais homogêneos, com economia de energia e tempo. Dentre estas qualidades destaca-se o método de combustão em solução utilizando os nitratos dos respectivos metais e um combustível, como glicina ${ }^{(10,11)}$, sacarose, ácido cítrico ${ }^{(12)}$, uréia ou outros combustíveis orgânicos, onde a reação de combustão fornece a energia necessária para a formação dos precursores ${ }^{(1)}$.

A energia emitida para a reação redox é o que determina a morfologia dos cristais formados ${ }^{(12)}$, onde a alta energia de ignição, produção de gases e exotermicidade são variáveis dependentes do tipo de combustíveis utilizados e razão combustível/oxidante ${ }^{(1)}$. Para formação de aluminatos nanoestruturados utiliza-se o combustível glicina, em que a reação acontece lentamente com evolução dos gases que dissipam o calor formando grãos com tamanho em média de $30-55 \mathrm{~nm}^{(1,11,13,14)}$.

O tamanho dos cristais obtidos pode ser calculado por meio das imagens de microscopia eletrônica de transmissão e microscopia eletrônica de varredura e através da equação de Scherrer na caracterização da composição estrutural por difração de raios X.

No presente trabalho o objetivo é a obtenção de aluminato de cálcio na estrutura espinélio puro e dopado com cério, com economia de energia e tempo por meio da síntese através do método combustão em solução e tamanhos de cristais próximos ao nanométrico.

\section{MATERIAL E MÉTODOS}

O aluminato de cálcio é obtido através da mistura de soluções aquosas saturadas. O procedimento para obtenção das soluções é a adição do reagente em um béquer de $250 \mathrm{ml}$ e acréscimo de água destilada em quantidade mínima, apenas para dissolução. Utilizaram-se nitrato de alumínio nonahidratado (dinâmica, P.A- 98\%), nitrato de cálcio tetrahidratado (CETUS, P.A - 98\%) e glicina (Vetec, 98,5\%, $\mathrm{C}_{2} \mathrm{H}_{5} \mathrm{NO}_{2}$ ) ou sacarose $\left(\mathrm{C}_{12} \mathrm{H}_{22} \mathrm{O}_{11}\right)$. A seguir, todas as soluções são misturadas em um único béquer e aquecidas em chapa de aquecimento com agitação magnética até a ignição em $200^{\circ} \mathrm{C}$. Realizou-se o procedimento nas razões de combustível/oxidantes $\varphi$ de $(1,0$ e 2,5$)$ correspondente a estequiometria da reação e acima da estequiometria de acordo a Eq. 1:

$$
\begin{aligned}
& \varphi=\mathrm{n}\left\{2 \cdot 4 \mathrm{~N}_{\mathrm{C}}+5 \cdot 1 \mathrm{~N}_{\mathrm{H}}+1 \cdot 0 \mathrm{~N}_{\mathrm{N}}+2 \cdot(-2) \mathrm{N}_{\mathrm{O}}\right\} / \mathrm{x}\left\{1 \cdot 2 \mathrm{~N}_{\mathrm{Ca}}+\right. \\
& \left.+2\left[1.0 \mathrm{~N}_{\mathrm{N}}+3 \cdot(-2) \mathrm{N}_{\mathrm{O}}\right]\right\}+\mathrm{x}\left\{1 \cdot 3 \mathrm{~N}_{\mathrm{Al}}+3 \cdot\left[1 \cdot 0 \mathrm{~N}_{\mathrm{N}}+3 \cdot(-2) \mathrm{N}_{\mathrm{O}}\right]\right\}
\end{aligned}
$$


onde n é a quantidade em mols do combustível glicina ou sacarose, $\mathrm{x}$ é a quantidade em mols do nitrato de cálcio e alumínio e quando $\varphi=1$ a reação é estequiométrica e $\varphi>1$ a reação é rica em combustível, $\mathrm{N}_{\mathrm{i}}$ representa o número de cada átomo nos compostos.

Nesta pesquisa foram utilizadas as proporções molares nitrato de cálcio, alumínio e glicina de 1:2:4,5 e 1:2:5,5 e com sacarose proporções de 1:2:0,8 e 1:2:2. Os precursores obtidos são calcinados em forno mufla da Quimis (Q318S24) com taxa de aquecimento de $20{ }^{\circ} \mathrm{C} / \mathrm{min}$ a $800{ }^{\circ} \mathrm{C}$ por $2 \mathrm{~h}$; tal processo é mostrado na Fig. 2.

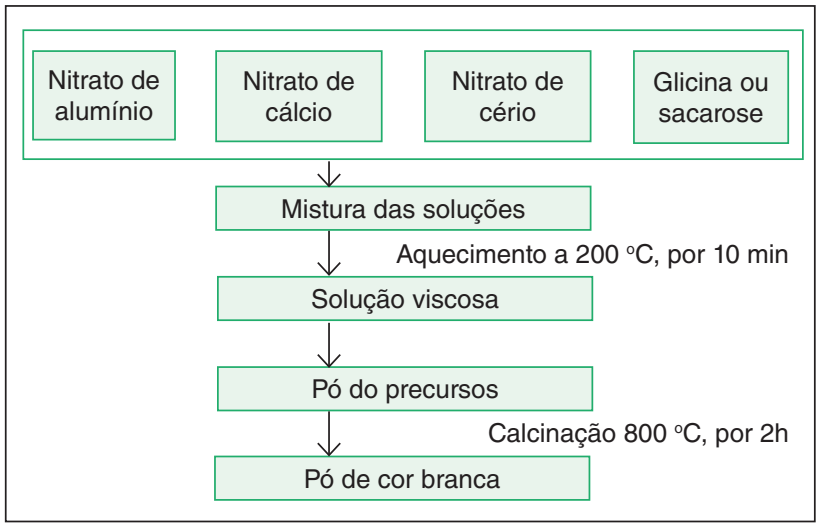

Figura 2: Síntese de aluminato de cálcio puro e dopado com cério através do método combustão em solução.

Os materiais dopados foram sintetizados com adição da solução de nitrato de cério $\left(\mathrm{Ce}\left(\mathrm{NO}_{3}\right)_{3}\right)$ compondo a proporção dos nitrato de cério, cálcio, alumínio e glicina de 0,01:0,99:2:5,5 e 0,005:0,995:2:5,5 e quando utilizou-se sacarose nas mesmas proporções da dopagem mas com a quantidade de 2 mols em relação a quantidade de combustível. Esses precursores foram calcinados ao mesmo tempo em forno mufla da Quimis (Q318S24) com taxa de aquecimento de $20{ }^{\circ} \mathrm{C} / \mathrm{min}$ a $800{ }^{\circ} \mathrm{C}$ por $2 \mathrm{~h}$.

A morfologia dos precursores formados foi caracterizada por microscopia eletrônica de transmissão. Para o preparo da amostra, utilizou-se uma gota das amostras calcinadas dispersas em água destilada em ultrassom por $30 \mathrm{~min}$ depositada na grade de cobre. Por microscopia eletrônica de varredura, uma pequena quantidade do pó calcinado foi depositada sobre fita de carbono e metalizada com ouro para torná-la condutora. O equipamento utilizado foi o JSM - 5900 Jeol, acoplado ao EDS Vantage, Thermo/Noran Instruments, onde também foram obtidos os dados de composição do material.

A composição estrutural das amostras calcinadas de aluminato de cálcio puras foi obtida por difratogramas de raios $\mathrm{X}$, através do equipamento de difração de raios X da Philips, monocromador de grafite e ânodo de cobre, gerador PW-1830, controlador de difratômetro PW - 1840. O equipamento foi regulado para a medida do ângulo $2 \theta$ com varredura de 10 à $70^{\circ}$ com passo de $0,02^{\circ}$ e contagem de 4 segundos para cada ângulo.

\section{RESULTADOS E DISCUSSÃO}

As reações redox são normalmente exotérmicas e não há um controle em relação a variáveis como temperatura e evolução de gases, para induzir determinadas características desejáveis como morfologia e cristalografia do material foram utilizados combustíveis com características diferentes, como a glicina que possui uma temperatura de ignição menor e a reação não ocorre de maneira violenta comparada à sacarose, neste caso quando a reação ocorre lentamente os cristais são menores devido a temperatura manter a reação entre os reagentes e não formar aglomerados. Quando, utilizam-se proporções molares de combustíveis diferentes há variação da temperatura de ignição o que pode ocasionar a formação de maior quantidade de gases, como também diminui o tempo de reação.

\section{Difração de raios $X(X R D)$}

A composição do material foi identificada de acordo com picos obtidos dos difratogramas. Através dos gráficos é observado que há diferença nas estruturas obtidas com a variação da proporção molar da glicina. Com destaque para os ângulos $2 \theta$ de maior intensidade em $37^{\circ}, 44^{\circ}$ e $65^{\circ}$ referente a estrutura predominante da fase $\mathrm{CaAl}_{2} \mathrm{O}_{4}$ e mistura com a fase $\mathrm{Ca}_{2} \mathrm{Al}_{3} \mathrm{O}_{6}{ }^{(7)}$, segundo comparação do banco de dados da American Mineralogist Crystal Structure (AMSC) e de acordo ao estudo de Lakshmanan ${ }^{(15)}$. Observou-se nos difratogramas da Fig. 3 uma uniformidade dentre as fases das amostras obtidas através das diferentes proporções de glicina e sacarose, sendo caracterizada uma das fases como o espinélio de aluminato de cálcio. $\mathrm{Na}$ amostra com quantidade de glicina estequiométrica nota-se a composição de uma quantidade maior de misturas das fases $\mathrm{Ca}_{3} \mathrm{Al}_{2} \mathrm{O}_{6}, \mathrm{CaAl}_{2} \mathrm{O}_{4}$, $\mathrm{CaAl}_{4} \mathrm{O}_{7}$ e $\mathrm{CaAl}_{12} \mathrm{O}_{19}$ a banda identificada entre $30^{\circ}$ e $40^{\circ}$, são

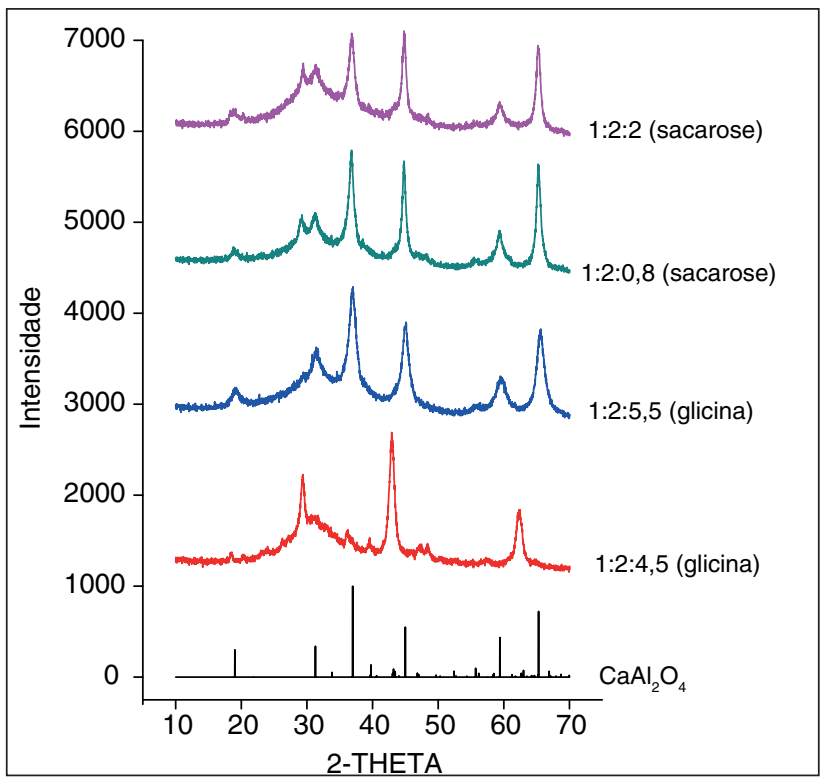

Figura 3: Difratogramas das amostras de aluminato de cálcio puras em vermelho 1:2:4,5 (glicina), azul 1:2:5,5 (glicina), verde $1: 2: 0,8$ (sacarose), rosa 1:2:2 (sacarose) e em preto dados da AMSC da estrutura $\mathrm{CaAl}_{2} \mathrm{O}_{4}$. 
compostos binários do aluminato que em alta temperaturas reagem e predomina a fase $\mathrm{CaAl}_{2} \mathrm{O}_{4}$, como encontrado nas outras amostras. A diferença ocorreu nas amostras de XRD devido à variação da temperatura de reação, quando pela alta temperatura na reação a fase espinélio é predominante.

A morfologia dos cristalitos formados foi obtida por meio dos dados dos cinco picos mais intensos do difratograma de acordo a Tabela 1 onde não houve variação do tamanho dos cristais formados, obtendo-se como resultado que a diferença de temperatura adquirida por meio da variação da proporção de combustível está relacionada a constituição de fase do material. O menor tamanho obtido através do combustível glicina não está relacionado à aglomeração dos cristais devido a evolução mais lenta da reação.

Tabela 1: Tamanho do cristalito calculado pela equação de Scherrer dos cinco picos de Bragg mais intensos e média dos tamanhos encontrados.

\begin{tabular}{c|c|c|c|c|c|c|}
$\begin{array}{c}\text { Ca:Al: } \\
\text { combustível } \\
(\mathbf{m o l})\end{array}$ & $\begin{array}{c}\mathbf{D}_{1} \\
(\mu \mathrm{m})\end{array}$ & $\begin{array}{c}\mathbf{D}_{2} \\
(\mu \mathrm{m})\end{array}$ & $\begin{array}{c}\mathbf{D}_{3} \\
(\mu \mathrm{m})\end{array}$ & $\begin{array}{c}\mathbf{D}_{4} \\
(\mu \mathrm{m})\end{array}$ & $\begin{array}{c}\mathbf{D}_{5} \\
(\mu \mathrm{m})\end{array}$ & $\begin{array}{c}\text { Média } \\
(\mu \mathrm{m})\end{array}$ \\
\hline $1: 2: 4,5$ & 0,23 & 0,28 & 0,12 & 0,32 & 0,17 & 0,22 \\
\hline $1: 2: 5,5$ & 0,11 & 0,09 & 0,14 & 0,21 & 0,14 & 0,13 \\
\hline $1: 2: 0,8$ & 0,15 & 0,41 & 0,29 & 0,19 & 0,32 & 0,27 \\
\hline $1: 2: 2$ & 0,17 & 0,17 & 0,26 & 0,13 & 0,36 & 0,21 \\
\hline
\end{tabular}

\section{Microscopia eletrônica de transmissão (MET)}

$\mathrm{O}$ tamanho das partículas e a morfologia do $\mathrm{CaAl}_{2} \mathrm{O}_{4}$ foram obtidos através das imagens geradas pelo equipamento de MET, onde se pode observar uma formação de cristais em um aglomerado, na Fig. 4 (A), e outros separados, com a formação individual definida em cubo, na Fig. 4 (B), quando utilizado o combustível glicina em diferentes proporções.

A morfologia de organização dos cristais em aglomerado e isolados está de acordo a quantidade de gases gerados na combustão, uma vez que a maior quantidade de gases liberados, devido à maior quantidade de combustível na reação, separa os grãos e a evolução mais lenta da reação evita a dissipação rápida do calor necessário para formação da fase espinélio e obtenção de cristais menores. Consequentemente, quando há aumento da quantidade molar do combustível na reação os cristais cerâmicos formados foram menores de acordo as Tabelas 1 e 2 .

Tabela 2: Descrição do tamanho das imagens de MET dos aluminatos de cálcio

\begin{tabular}{c|c|c|}
$\begin{array}{c}\text { Razão } \\
\text { Ca:Al:combustível } \\
(\text { mol })\end{array}$ & $\begin{array}{c}\text { Tamanho médio } \\
\text { de grãos } \\
(\mu \mathrm{m})\end{array}$ & $\begin{array}{c}\text { Característica } \\
\text { morfológica }\end{array}$ \\
\hline $1: 2: 4,5$ & 0,34 & Aglomerados \\
\hline $1: 2: 5,5$ & 0,12 & $\begin{array}{c}\text { Cristais mais } \\
\text { simples }\end{array}$ \\
\hline $1: 2: 0,8$ & 0,11 & Aglomerados \\
\hline $1: 2: 2$ & 0,44 & Cristais separados \\
\hline
\end{tabular}

Na Fig. 5(A) está a imagem MET da amostra produzida com sacarose e pode-se observar que há uma quantidade de material na forma de cristais mais aglomerados e outra na forma de finas linhas de cristais caracterizando menores grãos de acordo a Tab.2. Não foram obtidos cristais isolados. Essas formações podem ser devido à maior rapidez da reação que promoveu a dissipação do calor na combustão da sacarose com menor proporção de combustível. Na Fig. 5(B), observou-se apenas a formação de

A

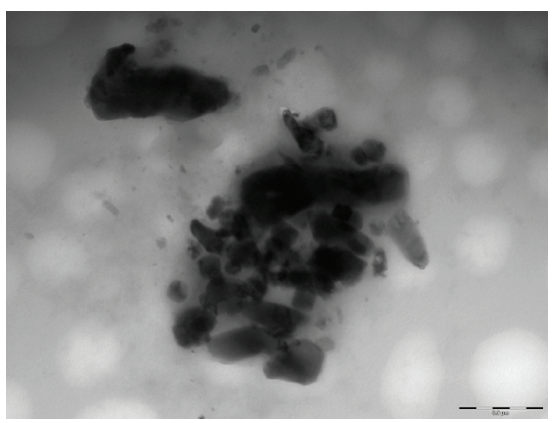

B

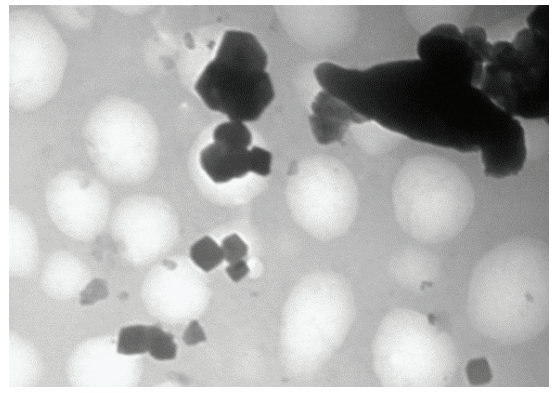

Figura 4: Imagem de MET do CaAl2O4, da síntese com razões de combustível glicina /oxidantes (A) $\phi=1,0$ e (B) $\phi=2,5$.

A

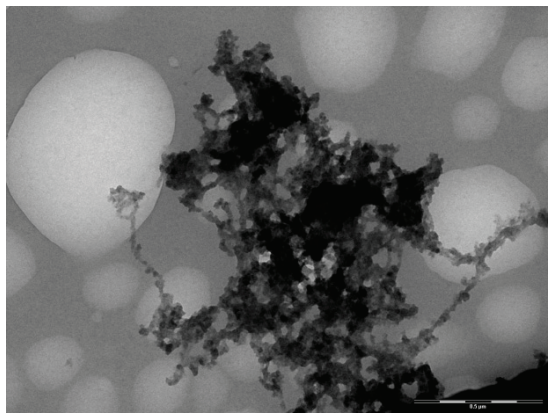

B

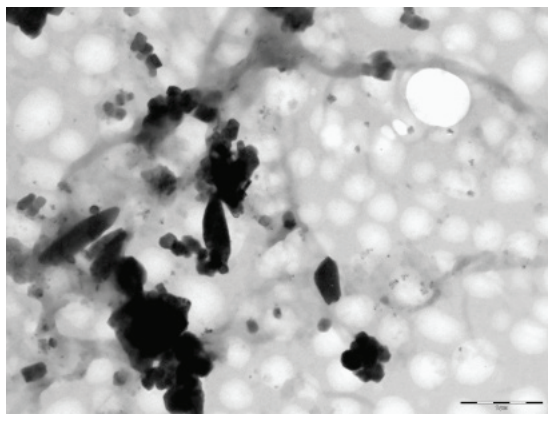

Figura 5: Imagem de MET do $\mathrm{CaAl}_{2} \mathrm{O}_{4}$, da síntese com razões de combustível sacarose /oxidantes (A) $\phi=1,0$ e (B) $\phi=2,5$. 
material particulado agregado, mas, não a formação de finas linhas de cristais. Neste caso, a maior proporção de combustível deve ter promovido um considerável aumento na temperatura e também um maior tempo de combustão permitindo que as partículas formassem agregados maiores quando comparado ao uso de glicina nas mesmas condições, onde não houve a formação das linhas finas de partículas em nenhuma das concentrações. Esse fato poderia ser atribuído à reação de combustão ser mais rápida devido ao teor mais energético da sacarose, o que gerou grande quantidade de energia favorecendo pelo aumento significativo da temperatura a velocidade da reação e a aglomeração dos grãos. Em relação à diferença de proporção molar, o tamanho dos cristais aumentam em relação a glicina devido à maior temperatura de ignição que aumentam a rapidez da reação e a energia promove os cristais maiores e afastados e evita a coalescência do material.

\section{Microscopia eletrônica de varredura (MEV)}

Nas imagens de MEV observa-se que a superfície de formação das amostras de $\mathrm{CaAl}_{2} \mathrm{O}_{4}: 1 \% \mathrm{Ce}^{+3}, \mathrm{CaAl}_{2} \mathrm{O}_{4}: 0,5 \% \mathrm{Ce}^{+3}$ possui a aparência de bolhas e poros. Essa característica condiz com a evolução de gases durante a reação de combustão, onde na medida em que a combustão é mais rápida, maior a quantidade dos gases liberados e maior homogeneidade da superfície do material. Nas Figs. 6(A) e 6(B) observam-se tamanhos de bolhas menores e em toda superfície, pois na síntese foi usado como combustível a glicina, a reação de combustão ocorre mais lentamente, formando-se esferas menores.

Quando foi usada sacarose, com uma violenta evolução de gases, os tamanhos dos poros aumentaram consideravelmente, como evidenciado nas Figs. 7(A) e 7(B). A proporção molecular

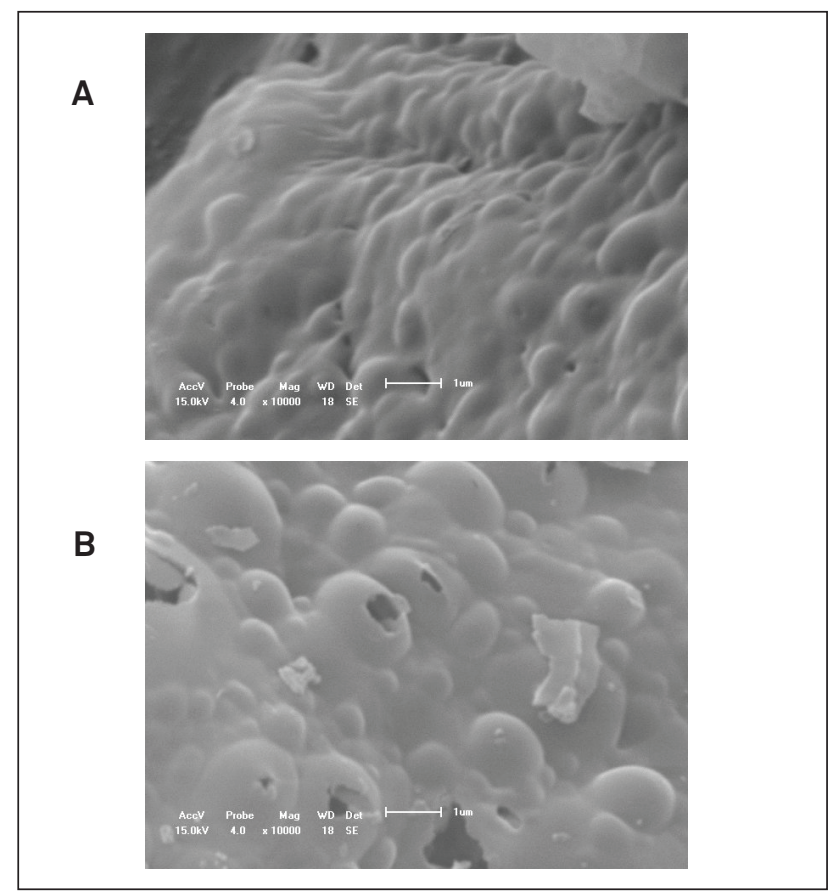

Figura 6: Imagem de SEM da amostra preparada com combustível glicina de (A) $\mathrm{CaAl}_{2} \mathrm{O}_{4}: 1 \% \mathrm{Ce}$ e (B) $\mathrm{CaAl}_{2} \mathrm{O}_{4}: 0,5 \% \mathrm{Ce}$. destes compostos com diferentes proporções de combustível e concentração de dopagem são equivalentes, como identificado através do espectro de EDX da Fig. 8, caracterizando-se como mesma composição de aluminato de cálcio.

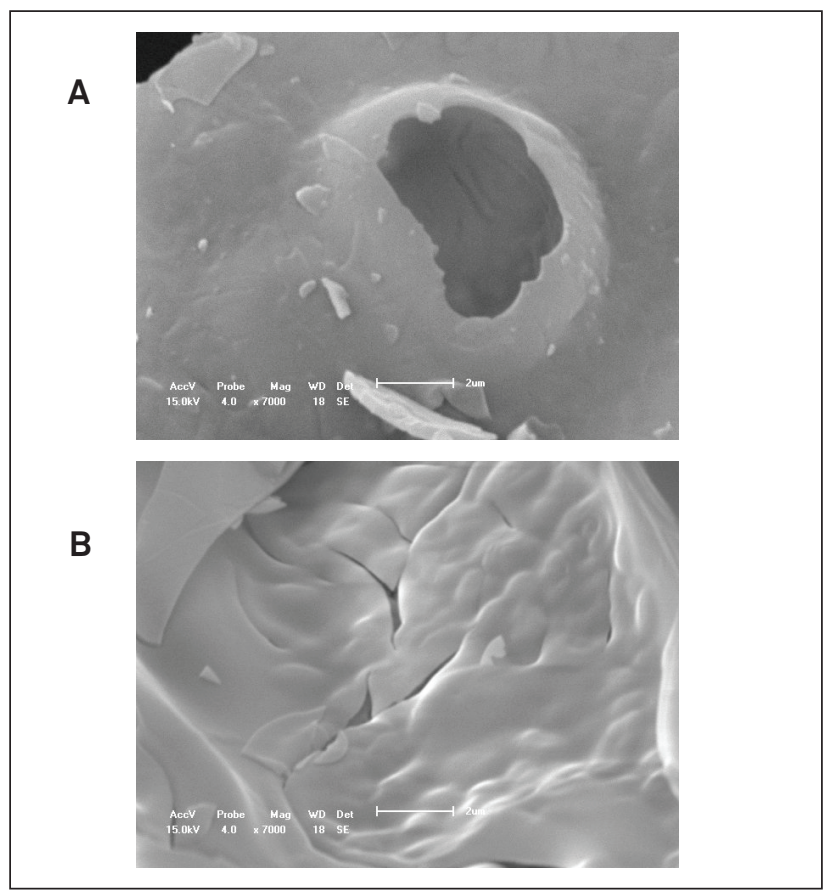

Figura 7: Imagem de SEM da amostra preparada com combustível sacarose de (A) $\mathrm{CaAl}_{2} \mathrm{O}_{4}: 1 \% \mathrm{Ce}$ e (B) $\mathrm{CaAl}_{2} \mathrm{O}_{4}: 0,5 \% \mathrm{Ce}$.

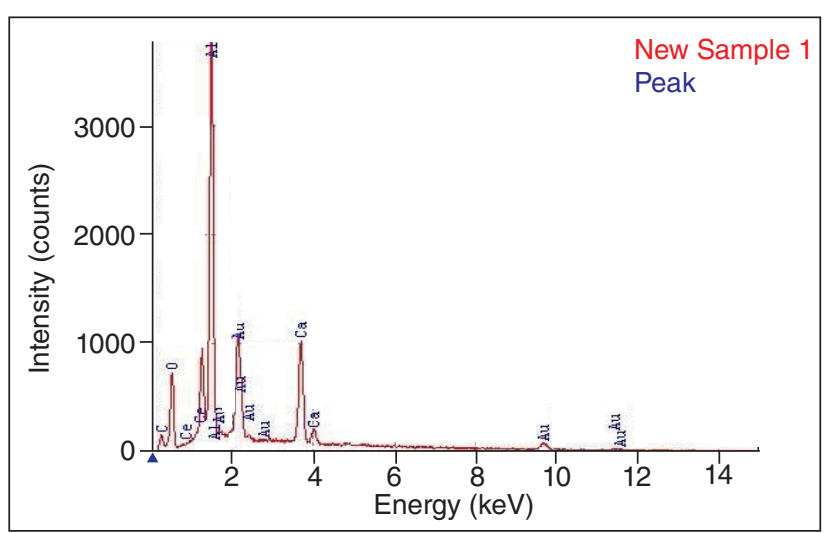

Figura 8: Espectro de EDX identificando a maior concentração do elemento alumínio, em seguida cálcio e cério.

\section{CONCLUSÃO}

Através do método combustão em solução é possível a obtenção de aluminatos de cálcio da série espinélio e mesmo com a dopagem do elemento Cério a proporção molecular não é modificada. Os tamanhos dos cristais dos compostos se diferenciam de acordo com a proporção combustível/oxidantes, onde pelo aumento da quantidade molar de combustível, na glicina de 4,5 para 5,5, o tamanho dos grãos diminuem o que não ocorre com a sacarose em que a amostra com proporção estequiométrica possui cristais menores que o não 
estequiométrico devido ao excesso de energia na reação. Nas amostras com síntese pura de aluminato de cálcio e dopadas com cério é observado que o uso de combustíveis diferentes não produz grandes variações no tamanho dos cristais do material, mas em relação à composição o uso de maior energia na reação diminui as outras composições de fases do aluminato para a predominância da fase espínelio.

\section{AGRADECIMENTOS}

Agradecimentos a Fundação de Amparo a Pesquisa do Estado da Bahia (FAPESB) pelo financiamento de bolsas de mestrado, ao Centro de Microscopia da UESC (CME) pela utilização do Microscópio de Transmissão e ao Instituto de Pesquisas Espaciais (INPE) pelas análises de difração de raios-X realizadas por Nila Lopes e prof. Dr. Danilo Barquete.

\section{REFERÊNCIAS}

1. PATIL, K. C., HEGDE, M. S., RATTAN, T., ARUNA, S. T. Nanocrystalline Oxide Materials Combustion Synthesis, Properties and Applications, World Scientific Publishing, London, 2008.

2. ZAWRAH, M. F., SHEHATA, A. B., KISHAR, E. A., YAMANI, R. A. Elsevier Masson SAS on behalf of Academie des sciences (2010),

3. GUIFANG, J., HU, Y., CHEN, L., WANG, X., Journal Applied Physics111, (2012), 113508-1
4. LIU, R. S., Phospors up conversion nano particles, quatum dots and their applications, Springer Science: volume 2, Taiwan, 2016. Disponível em: < https://books.google.com.br/books?id $=$ BYr2DAAAQBAJ\&pg=PA170\&lpg=PA170\&dq $>$, acesso em: $13 / 01 / 2017$

5. KODAMA, N., TANII, Y., YAMAGA, M. Jounal of luminescence 87, (2000), 1076.

6. MADHUKUMAR, K., BABU, K. R., PRASAD, K. C. A., JAMES, J., ELIAS, T. S., PADMANABHAN, V., NAIR, C. M. K., Bulletin of Materials Science 29, (2006), 119.

7. CHEN, W., WANG, Y., XU, X., ZENG, W., GONG, Y., ECS Solid State Letters1, (2012), R 17.

8. WANGN, Y., GONG, Y., XU, X., LI Y., Journal of Luminescence 133 (2013), 25.

9. SAMPAIO, J. A., Investigação de vidros de aluminato de cálcio dopado com íons terras raras. Dissertação de Mestrado (1997). Instituto de Física Gleb Wataghin, São Paulo (SP).

10. SUN, L., YAO, J., LIU, C., LIAO, C., YAN, C., Journal of Luminescence 87-89 (2000), 447.

11. CHAVAN, S.V., SASTRY, P. U. M., TYAGI, A. K. Journal of Alloys and Compounds 456 (2008) 51

12. GOMES, J., SERRA, O. A., PIRES, A. M., Química Nova 5, (2004), 706.

13. FU, Y. P., LIN, C. H., HSU, C. S., Journal of Alloys and Compounds 391, (2005), 110

14. PALNEEDI, H., MANGAM, V., DAS, S., DAS, K., Journal of Alloys and Compounds 509 (2011), 9912.

15. LAKSHMANAN, A., Sinterring of Ceramics, InTechWeb.org, Croatia, 2012. 